\title{
El G20 ante la pandemia del COVID-19: agenda, acciones y perspectivas
}

The G20 and the COVID-19 pandemic: agenda, actions and future perspectives

O G20 diante a pandemia do COVID-19: agenda, açôes e perspectivas

Leonardo Ramos ${ }^{1}$

Greta Rubinich ${ }^{2}$

DOI: $10.5752 /$ P.1809-6182.2020v17n3p13

Recibido el 23 de junio de 2020 Aprobado el 07 de agosto de 2020

\section{Resumen}

El objetivo es analizar el papel del G20 como comité de crisis frente la pandemia del COVID-19. Se describe su origen y funcionamiento para comprender las estructuras institucionales que condicionan su accionar. Se analizan las reuniones extraordinarias de Lideres $y$ de Ministros de Finanzas para enumerar las acciones que ha tomado hasta el momento.

Palabras Clave: G20. Comité de crisis. Respuesta multilateral.

\begin{abstract}
This paper aims to analyze the G20 as a crisis committee manager in the COVID-19 pandemic. Its origin and functioning are described, in order to understand the institutional structures that condition its actions. Extraordinary Meetings of Leaders and Finance Ministers are analyzed to list the actions the G20 has taken so far.

Keywords: G20. Crisis committee. Multilateralism.

\section{Resumo}

O objetivo é analisar o papel do G20 como comitê de crise diante à pandemia do COVID-19. São descritos seus origens e funcionamento para compreender as estruturas institucionais que condicionam suas açōes. As reunióes extraordinárias de Lideres e Ministros das Finanças são analisadas para listar as açôes que o grupo vem realizando até o momento.

Palavras chave: G20. Comitê de crise. Multilateralismo
\end{abstract}

\footnotetext{
1 Doctor en Relaciones Internacionales por la Pontifícia Universidade Católica do Rio de Janeiro (PUC-Rio), profesor asociado, Departamento de Relaciones Internacionales, Pontifícia Universidade Católica de Minas Gerais (PUC Minas). Belo Horizonte, Brasil. https://orcid.org/0000-0001-8245-6498

2 Maestranda en Relaciones Internacionales en Pontificia Universidade Católica de Minas Gerais (PUC-Minas) y becaria del Conselho Nacional de Desenvolvimento Cientifico e Tecnológico (CNPq). Licenciada en Relaciones Internacionales por la Universidad Nacional de Rosario (UNR). Belo Horizonte, Brasil. https://orcid.org/0000-0002-1425-8614
} 
Nuestro objetivo es analizar el rol del G20 ante la pandemia del COVID-19, más precisamente discutir el papel que asume frente a esta nueva crisis internacional, si actuando como comité de crisis (COOPER, 2010; MARTIN, 2013) o como foro para la gobernanza global (KIRTON, 2013). Ambas dualidades fueron resaltadas y analizadas por los especialistas a lo largo de estos 12 años de existencia del grupo, enfocándose en su evolución institucional y su inédita forma gobernanza interna (COOPER, 2019; LUCKHURST, 2016).Es por ello, que ante la nueva crisis sistémica originada por la pandemia del COVID-19, es importante analizar las acciones que el G20 ha tomado hasta el momento para intentar mitigar sus efectos económicos y sanitarios.

Los orígenes del G20 se remontan a la crisis asiática de 1999, cuando se suman al G7algunos de los países con mayor importancia sistémica de ese momento(ENGLISH; THAKUR; COOPER, 2005; MARTIN, 2013). Su objetivo inicial era comercial y financiero ya que del grupo participaban los Ministros de Economía y los Presidentes de los Bancos Centrales de: Alemania, Arabia Saudita, Argentina, Australia, Brasil, Canadá, China, Corea del Sur, Estados Unidos, Francia, India, Indonesia, Italia, Japón, México, Reino Unido, Sudáfrica y Turquía, junto con la Unión Europea.

En 2008, frente al colapso financiero internacional (MENEZES; RAMOS, 2018), los gobiernos de Estados Unidos y Francia promovieron una jerarquización del G20, convocando a una reunión de alto nivel. Era importante incluir en la mesa de discusiones a aquellos países que podían aportarle liquidez al sistema: los emergentes y los países en desarrollo, lo cual le otorgaba al grupo una mayor legitimidad para comandar la respuesta internacional frente a la crisis (RAMOS, 2014; SANAHUJA, 2012). Así, nace el G20 líderes compuesto por los Jefes y Jefas de Estado y de Gobierno de estos 19 países, más la Unión Europea.

Luego de coordinar una exitosa respuesta multilateral ante la crisis (KIRTON, 2013; MARTIN, 2013), el G20 reafirma su rol como comité de crisis (COOPER, 2010) y comienza a incursionar e importarse por otros temas sistémicos, tales como el desarrollo sostenible, medio ambiente, la lucha contra el crimen organizado transnacional y el terrorismo, la educación, la igualdad de género y el futuro del trabajo, entre otros (MERKE; ZACATTO, 2018), denotando su voluntad de erigirse como un importante foro para la gobernanza mundial (KIRTON, 2013).

Así, el grupo complejiza su funcionamiento, quedando compuesto por dos canales: el Canal de Finanzas, conformado por los ministros de Finanzas/Economía y los presidentes de los bancos centrales de los países miembros, y el Canal de Sherpas. El primero, se continúa ocupando de las reuniones y grupos de trabajo en materia económico-financiera; y el segundo, de las reuniones de los otros temas - más sociales y políticos. Los sherpas "guían" a los Jefes y Jefas de Estado y Gobierno, asesorándolos con respecto a los tópicos abordados en los distintos encuentros y grupos de trabajo (MERKE; ZACATTO, 2018).

Para 2020, Arabia Saudita había organizado una agenda para el grupo que fue completamente abandonada cuando la Organización Mundial de la Salud (OMS) declaró el brote del nuevo coronavirus SARS-CoV-2 como pandemia el 11 de Marzo de 2020. La nueva agenda del G20 pasó a ser la crisis sanitaria y la crisis económica que esta genera. Así, ha ocurrido una movilización multitemática de los paí- 
ses del G20 alrededor del nuevo coronavirus y sus efectos económicos-sanitarios en todas las reuniones del G20 (ministeriales, grupos de trabajo, grupos de afinidad).

Ante la urgencia de la crisis, Arabia Saudita invitó a los líderes del G20 a reunirse virtualmente el 26 de Marzo para organizar los ejes sobre los cuales el grupo trabajará para abordar la respuesta multilateral ante el COVID-19.En su reunión virtual extraordinaria ${ }^{3}$, los líderes se comprometieron a: "proteger vidas, salvaguardar a los trabajadores y sus ingresos, preservar la estabilidad financiera y promover su crecimiento y recuperación, minimizar las pérdidas de comercio global, brindar ayuda a los países que necesitan de asistencia y coordinar las políticas de salud pública y financieras" (G20 LIDERES, 2020). Estos objetivos colectivos son los ejes en los cuales se basa la nueva agenda para todas las reuniones del grupo de este año.

La Declaración de los Líderes del G20 contiene algunos puntos importantes que destacar. El primero, es el compromiso de inyectar recursos a los siguientes instrumentos público-privados: el Fondo de Respuesta Solidaria COVID-19 de la OMS, a la Coalición para las Innovaciones en Preparación para Epidemias (CEPI) y a la Alianza de Vacunas GAVI; y el llamamiento que realizan a toda la comunidad internacional para que donen a estos fondos, aportando recursos materiales ante la lucha contra el COVID-19. Con respecto a este punto, Arabia Saudita en su compromiso como presidente del grupo, declaró la donación de 500 millones de dólares (G20 PRESIDEN-

\footnotetext{
3 Todos los años, el presidente del grupo puede invitar a países no miembros a participar de las reuniones. Además, siempre se invita a representantes de los bloques regionales. También participan algunas organizaciones internacionales, y este año se destaca la presencia de la OMS.
}

CIA, 2020) y la contribución voluntaria hasta la fecha es de 21 billones de dólares ${ }^{4}$.

Otro punto a destacar es el Plan de Acción para combatir el COVID-19 que fue un pedido de los Líderes a los Ministros de Economía y Presidentes de los Bancos Centrales, es decir, es un pedido que se origina en el canal de los sherpas y que pasa al canal o track financiero. Este hecho denota que los Líderes dejan en manos de la acción de los grupos ministeriales o interministeriales, sobretodo de aquellos que pertenecen al track financiero, los compromisos que serán tomados para apaciguar los efectos económicos y sanitarios de la pandemia. El track financiero es el canal principal del grupo y es el que ha demostrado tener más avances en los últimos ańos, ya que cuenta con una instancia propia de monitoramiento y evaluación entre pares ${ }^{5}$ y con una trayectoria mayor (SCANDIUCCI, 2018).

Así, los ministros de Finanzas y Presidentes de Bancos Centrales, fueron los encargados de organizar la respuesta multilateral frente a la pandemia. En un primer momento, estos destacan su compromiso para con el intercambio continuo de datos oportunos, transparentes y estandarizados e información entre países (G20 FINANZAS, 2020) y refuerzan el pedido de donación de recursos materiales a los fondos público-privados anteriormente mencionados. Además, los ministros llaman a una próxima reunión conjunta con sus pares del área sanitaria, tal como fue expresamente encomendado por los líderes ${ }^{6}$. En un segundo momento,

4 Informaciones disponibles en: https://g20.org/en/Pages/ CombatCOVID19.aspx

5 El Consejo de Sustentabilidad Financiera (FSB, por sus siglas en inglés).

6 Esta innovación institucional de reuniones ministeriales conjuntas fue implementada por primera vez durante la presidencia de Japón en el año 2019 (KIRTON; WARREN, 2020) 
los ministros se comprometen a proporcionar liquidez y apoyo a las pequeńas y medianas empresas, así como a proteger los puestos de trabajo (G20 FINANZAS, 2020). Luego, los ministros reconocen que es primordial que se controle la propagación del virus para minimizar los efectos de la interrupción económica global y para generar la confianza necesaria al momento de reanudar la actividad (G20 FINANZAS, 2020).

Más adelante, los ministros reconocen el paquete estímulo de respuesta ante la crisis otorgado por el FMI, el cual amplia el monto de los préstamos de esta entidad, pero refuerzan la necesidad de que se busquen otras herramientas dentro de este organismo y de que se continúe con el proceso de reforma del mismo y de ajuste de las cuotas ${ }^{7}$.También, destacan el papel de ayuda de los Bancos de Desarrollo Regionales. Finalmente, los ministros deciden crear un fondo de infraestructura dentro del G20 para riesgos globales, incluida la prevención de futuras epidemias y pandemias (G20 FINANZAS, 2020).

Sin embargo, la decisión con mayor repercusión internacional de esta reunión fue el congelamiento de la deuda de los países del tercer mundo. Este compromiso, concede una moratoria de deuda bilateral oficial a las 76 economías más pobres del mundo. Estos son aquellos países considerados como destinatarios de la ayuda oficial al desarrollo (G20..., 2020) o los países menos desarrollados según la definición de ONU. Ya que la deuda privada o multilateral que tienen estos países es mayor que la bilateral, la sus-

7 La reforma de los organismos financieros multilaterales (FMI y BM) fue una de las acciones primordiales del grupo post crisis 2008, buscando que la participación de los países emergentes en los mismos, será mucho más relevante. Sobre este tema ver RAMOS, 2014. pensión de los pagos de la deuda bilateral desde mayo hasta diciembre de 2020 no generaría un importante efecto distributivo ni los ayudaría a combatir la pandemia con mayores recursos económicos (G20...,2020; IKOURIA, 2020) ${ }^{8}$.

Es por ello que los ministros de finanzas de los países africanos solicitaron la creación de un fondo de ayuda de emergencia de $\$ 100$ billones de dólares que les otorgue liquidez para afrontar la crisis económica y sanitaria, el cual sería otorgado a través del FMI (IKOURIA, 2020) Por otro lado, los países del Grupo Puebla también solicitaron al G20 una moratoria temporal de al menos un ańo del servicio de la deuda pública y privada, tanto de capital como de intereses a los países de ingreso medio y emergentes. Los beneficiarios serían: Colombia, México, Chile, Argentina e India, y también países desarrollados como España, Italia y Grecia (GRUPO PUEBLA, 2020).

Un último punto de repercusión internacional de ambas reuniones fue la creación del Global Response Pledging, fondo de $\$ 8$ billones de dólares para desarrollar la vacuna y los tratamientos médicos contra el COVID-19 (G20 PRESIDENCIA, 2020). De esta iniciativa participan sólo algunos países del G20, en su mayoría europeos (Francia, Alemania, Italia, Japón, Reino Unido, y la Unión Europea) junto Arabia Saudita (presidente del G20), Canadá y Noruega9. Aquí es interesante destacar el aislamiento de los Estados Unidos y la notoria ausencia de China, India, Rusia y Brasil, lo que afecta significativamente los procesos de cooperación en dicho tema y, por consiguiente, sus prospectos de éxito.

\footnotetext{
8 Para una discusión respecto a las deudas soberanas, ver FERNÁNDEZ ALONSO, 2019.

9 No es parte del G20 pero participa por su importante desarrollo farmacéutico.
} 
Los ministros de Salud del G20 también se reunieron virtualmente dejando un saldo negativo: no llegaron a firmar un comunicado oficial debido a la división en grupos (KIRTON, 2020). El único compromiso expresado en el documento que se envió a la prensa, luego de la reunión, fue que los ministros se reunirán nuevamente si así lo creen necesario. Aquí es importante destacar que, debido a la propia estructura interna del G20, la salud no es un tema de atención frecuente del grupo, y acaba que sea más probable que se lleguen a acuerdos en otras áreas relacionadas con el track de economía y finanzas, tales como comercio, inversiones, economía digital y turismo (CHODOR, 2020; LUCKHURST, 2016; SCANDIUCCI, 2018).

Tal como resalta Chodor (2020) aunque se haya dado una amplitud temática dentro del G20 desde que el grupo se autoreconoce como foro para la gobernanza global, el track de finanzas tiene un mayor peso a la hora de tomar decisiones comparado con los otros canales, por la antigüedad de su creación, en 1999, y por su procedimiento decisorio. Esto implica que el G20 hoy en día sea potencialmente más efectivo para tomar decisiones respecto a la crisis económica que genera la pandemia, y no para decidir respecto a las medidas sanitarias o de salud, cuyos lineamentos para la gestión de la pandemia siguen siendo los de la OMS - de ahí la importancia de la inclusión de la OMS en las conversaciones desde ahora.

Concluyendo, ante la pandemia del COVID-19 el G20 vuelve a su función de comité de crisis, y aparentemente con una relevancia renovada. Es el foro elegido para discutir la restructuración de la deuda, el financiamiento para una vacuna y las respuestas económicas-financieras para combatir la crisis sistémica global; dejando aún mucha expectativa para resolver sobre los temas sanitarios y de salud, sobretodo en lo que respecta al presupuesto permanente de la OMS, la cooperación en asistencia médica entre los países y la presencia o no del director de la OMS en la cumbre oficial en noviembre.

En un ańo plagado de incertidumbres, la pandemia viene a potenciar dinámicas ya existentes en el sistema internacional. El G20, contando con la participación de China, emerge como el grupo multilateral que ha tenido una mayor coordinación hasta el momento. Queda por ver cómo se darán los resultados de estos compromisos a lo largo de este año y de qué manera llegarán los líderes a su futura reunión en noviembre.

\section{Referencias}

CHODOR, Tom. Missing in action: The G20 in the Covid crisis. The Interpreter, [s.l.], Lowy Institute. 22 abr. 2020. Disponível em: https://www.lowyinstitute.org/the-interpreter/ missing-action-g20-covid-crisis. Acesso em: 22 jun. 2020

COOPER, A. F. The G20 as an improvised crisis committee and/or a contested 'steering committee' for the world. International Affairs, Oxford, v. 86, n. 3, p. 741-757, may. 2010.

COOPER, A. F. The G20 is dead as a crisis or steering committee: Long live the G20 as hybrid focal point. South African Journal of International Affairs, [s.l.], v. 26, n. 4, p. 505-520, 2019.

ENGLISH, J.; THAKUR, R.; COOPER, A. F. Reforming from the Top. A Leaders' 20 Summit. Hong Kong: United Nations University, 2005.

FERNÁNDEZ ALONSO, J. M. Crisis de deuda soberanas: aproximaciones en torno al debate contemporáneo de los mecanismos de reestructuración. Estudos Internacionais: revista de relaçóes internacionais da PUC Minas, Belo Horizonte, v. 7, n. 1, p. 7-24, abr. 2019.

G20 LÍDERES. Extraordinary G20 Leaders' Summit: Statement on COVID-19. Riyadh: Saudi G20 Presidency, 2020. Disponível em: http://www.g20.utoronto.ca/2020/2020-g20-statement-0326.html. Acesso em: 22 jun. 2020.

G20 PRESIDENCIA ARABIA SAUDITA. The Saudi G20 Presidency Calls for US\$8 Billion in Combatting the COVID-19 Pandemic: Saudi Arabia Pledges US\$500 Million. 2020. Disponível em: http://www.g20.utoronto. 
ca/2020/2020-g20-covid-funding-0416.html. Acesso em: 22 jun. 2020.

G20 PRESIDENCIA ARABIA SAUDITA. Saudi Arabia joins Coronavirus Global Response Pledging Event. Riyadh: Saudi G20 Presidency, 2020. Disponível em: http://www. g20.utoronto.ca/2020/2020-g20-pledging-0503.html. Acesso em: 22 jun. 2020.

G20 FINANZAS. Communiqué Virtual meeting of the G20 finance ministers and central bank governors. Riyadh: Saudi G20 Presidency, 2020. Disponível em: http://www.g20.utoronto.ca/2020/2020-g20-finance-0415.html. Acesso em: 22 jun. 2020

G20 debt relief plan no panacea for vulnerable emerging markets. Entrevistador: Rahul Ghosh. Entrevistada: Daniela Jayesuria.[S. I], Moody's Talks - Inside Emerging Markets Podcast. 27 Mayo 2020.

GRUPO PUEBLA. Grupo de puebla envía al G-20 propuesta de moratoria consensuada de la deuda para evitar una recesión global prolongada. 2020. Disponível em: https:// www.grupodepuebla.org/grupo-de-puebla-enviara-propuesta-economica-al-g-20-para-evitar-una-recesion-global-prolongada/. Acesso em: 22 jun. 2020.

IKOURIA, Edwin. The G20's promise of a debt freeze is not enough for Africa to combat the COVID-19 crisis. Euronews. -, p. 0-2. 20 abr. 2020. Disponível em: https://www.euronews.com/2020/04/20/g20-s-promise-of-a-debt-freeze-is-not-enough-for-africa-to-combat-the-covid-19-crisis-view. Acesso em: 22 jun. 2020.

KIRTON, J. J. G20 Governance for a Globalized World. Farnham: Ashgate, 2013.

KIRTON, John; WARREN, Brittaney. Selected Steps against a Comprehensive Threat: The G20 Leaders' Statement on
COVID-19. G20 Research Group, 2020. Disponível em: http://www.g20.utoronto.ca/analysis/200326-kirton-warren. html. Acesso em: 22 jun. 2020.

KIRTON, John. Disappointing Results from the G20 Health Ministers' Meeting.G20 Research Group,2020. Disponível em: http://www.g20.utoronto.ca/analysis/200419-kirton.html. Acesso em: 19 abr. 2020.

LUCKHURST, J. G20 Since the Global Crisis. New York: Palgrave Macmillan, 2016.

MARTIN, P. The G20: From Global Crisis Responder to Steering Committee. In: COOPER, A. F.; HEINE, J.; THAKUR, R. The Oxford Handbook of Modern Diplomacy. Oxford: Oxford University Press, 2013. Cap. 40.

MENEZES, R. G.; RAMOS, L. C. S. Apresentação - 10 anos da crise financeira (2008-2018): Leituras e interpretaçốes. Conjuntura internacional, Belo Horizonte, v. 15, n. 2, p. 1-2, sep. 2018.

MERKE, F.; ZACATTO, C. Introduccion: el G20 en un mundo incierto. Pensamiento propio, Buenos Aires, v. 23, p. 15-32, jul./dic. 2018.

RAMOS, L. Potências médias emergentes e reforma da arquitetura financeira mundial? Uma análise do BRICS no G20. Revista de Sociologia e Política, Curitiba, v. 22, n. 50, p. 49-65, jun. 2014.

SANAHUJA, J. A. El G-20 y la gobernanza economica global: cuestiones de representatividad, legitimidad y eficacia. Anuario Sociolaboral, Fundacion $1^{\circ}$ de Mayo, v. 02, p. 197-213, 2012.

SCANDIUCCI, J. G. F. o Brasil e o G20 (2008-2015). In: DESIDERÁ NETO, W. A.et al. Política externa brasileira em debate: dimensóes e estratégias de inserção internacional no pós-crise de 2008. Brasilia: Funag, 2018. cap. 6, p. 626. 